\title{
INFINITE GAMES WITH IMPERFECT INFORMATION $\left({ }^{1}\right)$
}

\author{
BY
}

\author{
MICHAEL ORKIN
}

\begin{abstract}
We consider an infinite, two person zero sum game played as follows: On the $n$th move, players $A, B$ select privately from fixed finite sets, $A_{n}$, $B$, the result of their selections being made known before the next selection is made. After an infinite number of selections, a point in the associated sequence space, $\Omega$, is produced upon which $B$ pays $A$ an amount determined by a payoff function defined on $\Omega$. In this paper we extend a result of Blackwell and show that if the payoff function is the indicator function of a set in the Boolean alyebra generated by the $G_{\delta}$ 's (with respect to a natural topology on $\Omega$ ) then the game in question has a value.
\end{abstract}

1. Introduction. Infinite games with imperfect information have been studied by several writers, notably Blackwell [1], [2], and Shapley [5]. Before proceeding with the main result of this paper, we will discuss the structure and admissible strategies of these games.

Let $\left\{A_{n}\right\},\left\{B_{n}\right\}$ be sequences of nonempty finite sets. Let $Z_{n}=A_{n} \times B_{n}$, and let $\Omega$ be the space $\Pi_{n=1}^{\infty} Z_{n}$ of infinite sequences $\omega=\left(z_{1}, z_{2}, \ldots\right)$ where $z_{n} \epsilon$ $Z_{n}$. Let $\Omega$ be topologized as follows (for a related discussion, see [3]):

Suppose $X$ is the set of all positions, i.e. finite sequences, $x=$ $\left(z_{1}, z_{2}, \cdots, z_{n}\right), z_{i} \in Z_{i}, n=0,1,2, \ldots$. Then if $\omega \in \Omega, x \in X$, we define $x$ to be a neighborhood of $\omega$ if $\omega$ passes through $x$. If the positions are thus considered as sets, they form a base for a Hausdorff, disconnected topology for $\Omega$ in which $\Omega$ is compact.

In this topology any open set is defined by a subset of $X$ (a countable collection of positions). Any set defined by a finite collection of positions is both open and closed. It is shown by Wolfe [7] that if $G$ is a $G_{\delta}$ then there exists a collection of positions $T$ such that $G=\{\omega \in \Omega \mid \omega$ passes through infinitely many members of $T$, which we will henceforth denote by $G=T$ i.o.

Now, suppose $f$ is a bounded Baire function on $\Omega$. Then we define a zero sum two person game $G_{f}$, played as follows:

Received by the editors July 1,1971 .

AMS 1970 subject classifications. Primary 90D05, 90D15; Secondary 28A05, 60G45.

Key words and phrases. Infinite games, imperfect information, two person zero sum game, lower value, payoff function, Baire function.

(1) This paper is part of the author's doctoral dissertation at the University of California, Berkeley, and was prepared with the partial support of the U. S. Air Force, Grant AF-AFOSR-1312-67, and the U. S. Office of Naval Research, Contract NONR N00014-66C0036. 
First, player $A$ selects $a_{1} \in A_{1}$ while player $B$ simultaneously selects $b_{1}$ $\in B_{1}$. The result, $z_{1}=\left(a_{1}, b_{1}\right) \in Z_{1}$, is announced to both players, upon which $A$ selects $a_{2} \in A_{2}$ while $B$ selects $b_{2} \in B_{2}$, etc. The result of this infinite sequence of moves is a point $\omega=\left(z_{1}, z_{2}, \ldots\right) \in \Omega$ and $B$ pays $A$ the amount $f(\omega)$.

A strategy $\alpha(\beta)$ for $A(B)$ gives for each position $x$ (of length $n$, say) a probability distribution on $A_{n+1}\left(B_{n+1}\right)$ with the stipulation that if the current position is $x, A(B)$ will make his next choice according to $\alpha(\beta)$. A pair of strategies $(\alpha, \beta)$ defines a probability distribution $P_{a \beta}$ on $\Omega$ and, hence, an expected payoff to $A$ in $G_{f}$ when $A$ uses $\alpha$ and $B$ uses $\beta$ :

$$
E(f, \alpha, \beta)=\int f(\omega) d P_{\alpha \beta}(\omega) .
$$

The lower and upper values of $G_{f}$ are, respectively,

$$
L\left(G_{f}\right)=\sup _{\alpha} \inf _{\beta} E(f, \alpha, \beta), \quad U\left(G_{f}\right)=\inf _{\beta} \sup _{\alpha} E(f, \alpha, \beta) .
$$

It is always true that $L\left(G_{f}\right) \leq U\left(G_{f}\right)$; if $L\left(G_{f}\right)=U\left(G_{f}\right)$, this common value is called the value of $G_{f}$ and will be denoted by $\operatorname{Val}\left(G_{f}\right)$.

2. Main Result. We will show that if $f=I_{G}$, where $G \in B\left(G_{\delta}\right)$ (the Boolean algebra generated by the $G_{\delta}^{\prime}$ 's), then $G_{f}$ has a value. In [1], Blackwell proved this result if $G$ is a $G_{\delta}$. Before proving this result, we give two examples of games of this type and mention a related open question.

Example 1. On each move, players $A$ and $B$ choose, simultaneously, a 0 or 1. The winning set $S$ of the form $G_{\delta} \cup F_{\sigma}$, is defined as follows:

$S=G \cup F$ where $G=\left\{\omega \mid \omega_{n}=(0,0)\right.$ for infinitely many $n$ and $\omega_{n}=(1,1)$ for infinitely many $n\}$,

$F=\left\{\omega \mid \omega_{n}=(0,0)\right.$ for at most finitely many $n$ and $\omega_{n}=(1,1)$ for at most finitely many $n\}$.

The value of this game is 1 , which can be achieved by $A$ with a nonrandom strategy; he starts by saying 1 on each move. If $B$ says 0 on every move, $F$ is hit. If $B$ ever says $1, A$ then starts saying 0 's. If $B$ then says 1 's forever, $F$ is still hit. If $B$ ever says 0 again, $A$ switches back to 1 's, etc. If there are an infinite number of changes $G$ is hit, otherwise $F$ is hit.

Example 2. The winning set is a $G_{\delta}$. On each move, the players choose simultaneously a 0 or 1 . If player $A$ ever says 1 , the game is over on that move; if $B$ also said $1, A$ wins; if $B$ said $0, B$ wins. If $A$ never says 1 , the game continues and $A$ wins if there are infinitely many moves with outcome $(0,0)$. (In other words, $A$ tries to predict $B$ 's choice. See [2] for a related game.)

The value of this game is 1 , but there are no optimal strategies for $A$. Here is a strategy for $A$, due to David Blackwell, which, for fixed $N$, guarantees $A$ at least $1-1 / N$ : 
Define $N_{j}=2^{j} N, j=1,2, \cdots$, so that

$$
\sum_{j=1}^{\infty} \frac{1}{N_{j}}=\frac{1}{N}
$$

Player $A$ divides the trials into successive blocks of lengths $N_{1}, N_{2}, \cdots$. If he has not yet stopped the game, i.e. played 1 when block $j$ is reached, he selects $X_{j}$ at random from $\left\{1,2, \cdots, N_{j}\right\}$. He then plays 1 at the $X_{j}$ th trial of block $j$ if $B$ 's previous $X_{j}-1$ plays in the block are all 1's; otherwise, he plays 0 throughout the block. Then, clearly,

$$
P(A \text { loses on } j \text { th block } \mid j \text { th block is reached }) \leq 1 / N_{j}
$$

Thus, $P(A$ loses on $j$ th block $) \leq 1 / N_{j}$, and $P(A$ loses by failing to match $) \leq$ $\Sigma\left(1 / N_{j}\right)=1 / N$. However, by the nature of this strategy, if the game goes on forever, $A$ will win, since there would then be $(0,0)$ 's in each block.

The following question remains unsolved in general: Do games with payoffs which are simple functions based on sets in $B\left(G_{\delta}\right)$ have a value, i.e. games with payoff of the form $f=c_{1} I_{B_{1}}+\cdots+c_{n} I_{B_{n}}$, where $B_{i} \in B\left(G_{\delta}\right), c_{i}$ are constants. In fact, we do not even know whether or not the much simpler games with payoffs of the form $I_{Q_{1}}-I_{Q_{2}}$ have a value, where $Q_{1}, Q_{2}$ are open and disjoint. In another paper we will discuss some special cases of these kinds of games and show that they have a value.

We are now ready to prove the main result.

Lemma 1. Consider the class of sets of the form

$$
G_{1} \cup F_{1} \cup\left(G_{2} \cap F_{2}\right) \cup \cdots \cup\left(G_{n} \cap F_{n}\right)
$$

where $G_{i} \in G_{\delta}, F_{i} \in F_{\sigma}$. This class of sets is precisely $B\left(G_{\delta}\right)$.

Proof. By the fact that a finite union of $G_{\delta}$ 's is a $G_{\delta}$, a finite intersection of $F_{\sigma}$ 's is an $F_{\sigma}$, and by the standard results for generating Boolean algebras (e.g. see [4, Proposition I. 2.2, p. 7]) it is easily shown that every set in $B\left(G_{\delta}\right)$ is of the form $\bigcup_{i=1}^{n}\left(G_{i} \cap F_{i}\right), G_{i} \in G_{\delta}, F_{i} \in F_{\sigma}$. Thus, every set in $B\left(G_{\delta}\right)$ is of the form

$$
\begin{aligned}
& \left(\bigcup_{i=1}^{n}\left(G_{i} \cap F_{i}\right)\right)^{c}=\bigcap_{i=1}^{n}\left(G_{i}^{c} \cup F_{i}^{c}\right) \\
& \quad=\left(\bigcap_{i=1}^{n} F_{i}^{c}\right) \cup\left(\bigcap_{i=1}^{n} G_{i}^{c}\right) \cup\left(\bigcup_{i=1}^{n}\left(F_{i}^{c} \cap\left(\bigcap_{j \neq i} G_{j}^{c}\right)\right)\right)
\end{aligned}
$$


which, again using the fact about finite unions (intersections) of $G_{\delta}$ 's ( $F_{\sigma}$ 's) is easily seen to be of the required form.

Lemma 2. Consider the class of sets $\mathcal{C}=\bigcup_{n=1}^{\infty} \mathcal{C}_{n}$ generated in the following way: $\mathcal{C}_{1}=G_{\delta}$; if $n>1, \mathcal{C}_{n}=$ sets of the form $G_{\delta} \cup A_{n-1}$, where $A_{n}=$ complements of sets in $C_{n}\left(e . g . \mathcal{C}_{2}=G_{\delta} \cup F_{\sigma}\right)$. We claim $\mathcal{C}_{=B}\left(G_{\delta}\right)$.

Proof. By using De Morgan's law and the fact that $G_{\delta} \cup G_{\delta}=G_{\delta}, F_{\sigma} \cap F_{\sigma}=$ $F_{\sigma}$, it is easily seen that $\mathcal{C}_{4}=$ sets of the form $G_{1} \cup F_{1} \cup\left(F_{2} \cap G_{2}\right), \mathcal{C}_{2 n}=$ sets of the form $G_{1} \cup F_{1} \cup\left(\bigcup_{i=2}^{n}\left(F_{i} \cap G_{i}\right)\right)$ which by Lemma 1 gives the sets in $B\left(G_{\delta}\right)$.

The next lemma is the first step in an induction which will yield the main result.

Lemma 3. Let $\phi$ be upper semicontinuous, $0 \leq \phi \leq 1$. Suppose $G \in G_{\delta}$. Then the game with payoff $\bar{\phi}=\min \left(\phi, I_{G}\right)$ has a value.

Proof. The first part of this proof and $A$ 's method of play is the same as in [1]. Suppose $G=T$ i.o., where $T$ is a collection of positions. For any position $x$, let $G_{x}^{*}$ be the game, starting from $x$ with payoff $U_{t}\left(G_{\bar{\phi}}\right)$ if $T$ is hit for the first time after $x$ at $t$, with payoff 0 if $T$ is never hit after $x$, where $U_{t}\left(G_{\bar{\phi}}\right)$ is the upper value of the original game starting from $t$. This payoff is lower semicontinuous, so, by [6], $G_{x}^{*}$ has a value and player $B$ has an optimal strategy. We claim $\operatorname{Val}\left(G_{x}^{*}\right) \geq U_{x}\left(G_{\bar{\phi}}\right)$; for fixed $\epsilon>0$, we present a strategy for $B$ starting from $x$ such that no matter what $A$ does, $E_{x}(\bar{\phi}) \leq \operatorname{Val}\left(G_{x}^{*}\right)+\epsilon$ :

Let $B$, starting from $x$, play optimally in $G_{x}^{*}$ until $T$ is hit for the first time after $x$, say at $t$. Then $B$ plays, starting from $t$, to keep $E_{t}(\bar{\phi}) \leq U_{t}\left(G_{\bar{\phi}}\right)+\epsilon$, so

$$
E_{x}(\bar{\phi})=\sum_{t \in T} p(t) E_{t}(\bar{\phi}) \leq \sum_{t \in T} p(t) U_{t}\left(G_{\bar{\phi}}\right)+\epsilon \leq \operatorname{Val}\left(G_{x}^{*}\right)+\epsilon .
$$

Now, for $\epsilon>0$, we describe a strategy for $A$ such that no matter what $B$ does, $E(\bar{\phi}) \geq U\left(G_{\bar{\phi}}\right)-\epsilon$, and the lemma will be proved. First, $A$ plays $\epsilon / 4$ optimally in $G_{e}^{*}\left(e\right.$ denotes the empty position). If $T$ is hit after $e$, say at $t_{1}, A$ then plays $\epsilon / 8$ optimally in $G_{t_{1}}^{*}$, etc. (If $T$ is hit for the $n$th time at $t_{n}, A$ then plays $\epsilon / 2^{n+1}$ optimally in $G_{t_{n}}^{*}$.) Let the resulting sequence of moves be denoted by $z=$ $\left(z_{1}, z_{2}, \ldots\right)$.

We define a sequence of random variables: $X_{0}=U\left(G_{\bar{\phi}}\right)$; for $k \geq 1, X_{k}=$ $U_{t_{k}}\left(G_{\bar{\phi}}\right)$ if $T$ was hit for the $k$ th time at $t_{k}, X_{k}=0$ if $T$ was hit less than $k$ times. Thus, we have

$$
E\left(X_{k} \mid X_{k-1}, \cdots, X_{0}\right) \geq X_{k-1}-\epsilon / 2^{k+1}
$$


This is obvious if $X_{k-1}=0$. If not, $T$ was hit for the $(k-1)$ st time at $t_{k-1}$, say, after which $A$ played $\epsilon / 2^{k+1}$ optimally in $G_{t_{k}}^{*}$ to get at least Val $\left(G_{t_{k}}^{*}\right)-\epsilon / 2^{k+1}$ $\geq X_{k-1}-\epsilon / 2^{k+1}$. Since the payoff in $G_{t_{k}}^{*}$ is $X_{k}$, (1) follows. Taking expectations on both sides, we get

$$
E\left(X_{k}\right) \geq E\left(X_{k-1}\right)-\epsilon / 2^{k+1} \Rightarrow E\left(X_{k}\right) \geq U(G \bar{\phi})-\epsilon / 2 .
$$

Now, by the definition of upper semicontinuity and the nature of the topology on $\Omega$, for every point $z=\left(z_{1}, z_{2}, \ldots\right)$ and every $\epsilon>0$, there exists $k$ such that any point $\omega=\left(\omega_{1}, \omega_{2}, \ldots\right)$ with $\omega_{i}=z_{i}$ for $i \leq k$ has the property

$$
\begin{aligned}
\phi(\omega) \leq \phi(z)+\epsilon / 2 & \Rightarrow \text { if } z \in G, \bar{\phi}(\omega) \leq \bar{\phi}(z)+\epsilon / 2 \\
& \Rightarrow(\text { still if } z \in G) U_{\left(z_{1}, \cdots, z_{k}\right)}\left(G_{\bar{\phi}}\right) \leq \bar{\phi}(z)+\epsilon / 2 \\
& \Rightarrow \text { (for any } z \text { ) lim } \sup _{n} X_{n} \leq \bar{\phi}(z)+\epsilon / 2 .
\end{aligned}
$$

The last implication is obvious if $z \in G$. If $z \notin G, T$ is only hit say $N$ times, so for $n \geq N, X_{n}=0$. Using Fatou's lemma on the last inequality, we get

$$
E(\bar{\phi}) \geq \lim \sup _{n} E\left(X_{n}\right)-\epsilon / 2 \geq U\left(G_{\bar{\phi}}\right)-\epsilon .
$$

Theorem 1. Suppose $H \in \mathcal{C}_{n}$, i.e. $H=G \cup S, G \in G_{\delta}, S^{c} \in \mathcal{C}_{n-1}$. Suppose, also, that $\phi$ is upper semicontinuous with the property $0 \leq \phi \leq 1, \bar{\phi}=1$ on $S$. Then the game with payoff $\bar{\phi}=\min \left(\phi, I_{H}\right)$ bas a value.

Proof. Lemma 3 shows that the theorem is true for sets in $\mathcal{C}_{1}$. Suppose the theorem is true for sets in $\mathcal{C}_{n-1}$. Let $H \in \mathcal{C}_{n}, H=G \cup S$, where $G \in G \delta_{\delta}, S^{c} \epsilon$ $C_{n-1}$ (assume, wi thout loss of generality, that $G \neq \emptyset$ ). Suppose $G=T$ i.o. for some collection of positions $T$. For any position $x$, let $H_{x}^{*}$ be the game starting at $x$ which continues until the first time $T$ is hit after $x$, say at $t$, with $A$ getting $U_{t}\left(G_{\bar{\phi}}\right)$ when this happens. Otherwise, the game continues and $A$ gets $I_{S}$. We claim $H_{x}^{*}$ has a value (the payoff in $H_{x}^{*}$ may be neither upper nor lower semicontinuous).

Observe that if $C$ is closed, $J \in G_{\delta} \Rightarrow C \cap J \in G_{\delta} ; J \in F_{\sigma} \Rightarrow C \cap J \in F_{\sigma}$; therefore $J \in \mathcal{C}_{n} \Rightarrow C \cap J \in \mathcal{C}_{n}$. Let $\mathcal{O}_{x}$ be the open set defined by the collection of positions passing through $x$ which later hit $T$. Let $C_{x}=\mathcal{O}_{x}^{c}$. Then $S^{c} \cap C_{x} \epsilon$ $\mathcal{C}_{n-1}$ since $S^{c}$ is. Define $\phi^{*}=1-f$, where $f$ is the payoff in $H_{x}^{*}$. Also, define the upper semicontinuous function $g: g=\phi^{*}$ on $\Theta_{x}, g \equiv 1$ elsewhere. Thus $g$ satisfies the conditions of the theorem and $\phi^{*}=\min \left(g, I_{C_{x} \cap 5 c}\right)$, so by the induction hypothesis the game starting at $x$ with payoff $\phi^{*}$ has a value. Therefore, $H_{x}^{*}$ has a value since its payoff is $f=1-\phi^{*}$ (the method of proof in Lemma 3 allows negation of the payoff since the same proof can be used by reversing the role of the players; it clearly allows the addition of a constant to the payoff). 
By reasoning similar to that in Lemma 3 , it can be shown that $\operatorname{Val}\left(H_{x}^{*}\right) \geq$ $U_{x}\left(G_{\bar{\phi}}\right)$. Now, for fixed $\epsilon>0$, we will exhibit a strategy for player $A$ which guarantees that $E(\bar{\phi}) \geq U\left(G_{\bar{\phi}}\right)-\epsilon$.

As in Lemma 3, A starts out playing $\epsilon / 4$ optimally in $H_{e}^{*}$, etc. (If $T$ is hit for the $n$th time at $t_{n}, A$ then plays $\epsilon / 2^{n+1}$ optimally in $H_{t_{n}}^{*}$.) Let the resulting play be $z=\left(z_{1}, z_{2}, \cdots\right)$.

Define the random variables: $X_{0}=U\left(G_{\bar{\phi}}\right)$; for $k \geq 1, X_{k}=U_{t_{k}}\left(G_{\bar{\phi}}\right)$ if $T$ is hit for the $k$ th time at $t_{k}, X_{k}=I_{S}$ if $T$ is hit less than $k$ times. By reasoning similar to that in Lemma 3, we get

$$
E\left(X_{k}\right) \geq U\left(G_{\bar{\phi}}\right)-\epsilon / 2 \text {. }
$$

Again, by the definition of upper semicontinuity, there exists $k_{(\epsilon, z)}$ such that any point $\omega=\left(\omega_{1}, \omega_{2}, \ldots\right)$ agreeing with $z$ up to $z_{k(\epsilon, z)}$ has the property

$$
\begin{aligned}
\phi(\omega) \leq \phi(z)+\epsilon / 2 & \Rightarrow \text { if } z \in H, \bar{\phi}(\omega) \leq \bar{\phi}(z)+\epsilon / 2 \\
& \Rightarrow \text { if } z \in H, U_{\left(z_{1}, \cdots, z_{k}\right)}(G \bar{\phi}) \leq \bar{\phi}(z)+\epsilon / 2 \\
& \Rightarrow \text { for any } z, \lim \sup _{n} X_{n} \leq \bar{\phi}(z)+\epsilon / 2 .
\end{aligned}
$$

Again, the last step is obvious if $z \in G$. If not, $S \cap G^{c}$ is hit and $\bar{\phi}=1$ or $S^{c} \cap$ $G^{c}$ is hit and $\lim \sup _{n} X_{n}=0$. Thus, by Fatou, $E(\bar{\phi}) \geq \lim \sup _{n} E\left(X_{n}\right) \geq U\left(G_{\bar{\phi}}\right)-$ $\epsilon$.

Corollary 1. If $H \in B\left(G_{\delta}\right)$, the game with payoff $I_{H}$ has a value.

Proof. Let $\phi=1$ and use the theorem and Lemma 2.

Corollary 2. $G_{f}$ has a value if $f$ satisfies the following conditions:

(a) There is a collection $T$ of nonoverlapping positions (nonoverlapping means $x \in T \Rightarrow x$ is not an initial segment of any other member of $T$ ) such that if $x \in T$ then $f$ is constant on all sequences passing through $x$.

(b) $0 \leq f \leq 1$ on $T$.

(c) There exists $H \in B\left(G_{\delta}\right)$ such that $f=I_{H}$ if $T$ is never bit.

Proof. The function $\phi=1$ off $T, \phi=f$ otherwise, is upper semicontinuous, $\phi=1$ on $H$, and $f=\min \left(\phi, I_{H}\right)$ so the theorem applies.

Acknowledgement. I wish to express my appreciation to Professor David Blackwell for suggesting the problem herein and for his encouragement and advice during the course of my research.

\section{REFERENCES}

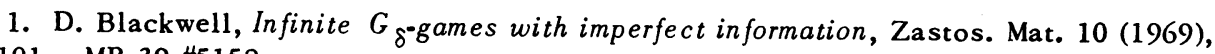
99-101. MR 39 \#5158. 
2. D. Blackwell and T. S. Ferguson, The big match, Ann. Math. Statist. 39 (1968), 159-163. MR 36 \#6211.

3. D. Gale and F. M. Stewart, Infinite games with perfect information, Contributions to the Theory of Games, vol. 2, Ann. of Math. Studies, no. 28, Princeton Univ. Press, Princeton, N. J., 1953, pp. 245-266. MR 14, 999.

4. J. Neveu, Bases mathématiques du calcul des probabilités, Masson, Paris, 1964; English transl., Holden-Day, San Francisco, Calif., 1965. MR 33 \#6659; \#6660.

5. L. S. Shapley, Stochastic games, Proc. Nat. Acad. Sci. U. S. A. 39 (1953), 10951100. MR 15, 887.

6. M. Sion, On general minimax theorems, Pacific J. Math. 8 (1958), 171-176. MR 20 \#3506.

7. P. Wolfe, The strict determinateness of certain infinite games, Pacific J. Math. 5 (1955), 841-847. MR 17, 506.

DEPARTMENT OF STATISTICS, UNIVERSITY OF CALIFORNIA, BERKELEY, CALIFORNIA 94720

Current address: Department of Mathematics, Case Western Reserve University, Cleveland, Ohio 44106 